\title{
Aspects of beyond standard model physics at the LHeC
}

\section{Georges Azuelos ${ }^{* \dagger}$}

TRIUMF/Université de Montréal

E-mail: georges.azuelos@umontreal.ca

\begin{abstract}
A brief overview is given here of some recent and preliminary studies on the potential of the $\mathrm{LHeC}$ for probing exotic physics processes. The $\mathrm{LHeC}$ can extend the exploration reach of many Beyond-Standard-Model theories expected to be explored at the LHC and can eventually provide a better understanding of their parameters. In particular, prospects for discovery or exclusion limits in the search for leptoquarks, heavy and excited fermions, contact interactions and diquarks are discussed.
\end{abstract}

XVIII International Workshop on Deep-Inelastic Scattering and Related Subjects April 19 -23, 2010

Convitto della Calza, Firenze, Italy

\footnotetext{
*Speaker.

${ }^{\dagger}$ representing the $\mathrm{LHeC}$ working group
} 


\section{Introduction}

In a few years, the LHC will hopefully have provided us with a good perspective of the physics that lies at the $\mathrm{TeV}$ scale. Although the proton collider's discovery reach for new physics beyond the Standard Model (BSM) is high, it will not always allow a precise measurement of the parameters of the new theories. The $\mathrm{LHeC}$ [1], as a possible successor to the LHC, has not only a rich physics programme in QCD and proton structure functions, but it will also allow to probe in more detail many BSM theories, thanks to a cleaner running environment, but also thanks to the possibility of measuring new observables, not available at the LHC. Here, we review briefly some recent studies on the $\mathrm{LHeC}$ potential for some BSM physics processes.

\section{Leptoquarks}

The remarkable relation between leptons and quarks in the Standard Model (SM), in their electromagnetic and weak interactions and their anomaly cancellations in the family structure, strongly suggests a fundamental connection. It is not surprising, therefore, that many BSM theories predict the existence of leptoquarks (LQ), particles that carry both lepton and baryon quantum numbers: extended gauge groups such as $E_{6}$ [2] where new fields can mediate interactions between leptons and quarks; extended technicolor [3, 4], where LQ's result from bound states of technifermions; the Pati-Salam model [5], where the leptonic quantum number is a fourth color of the quarks; lepton-quark compositeness models, or even supersymmetry, where LQ's are just a representation of squarks which decay via an R-parity violating interaction.

If they exist, leptoquarks will be abundantly produced at the $L H C$ in pairs [6], via $q q \rightarrow L Q L Q$ or $g g \rightarrow L Q L Q$, or singly via $g q \rightarrow \ell L Q$. Single production is suppressed by the $L Q-q-\ell$ coupling $\lambda_{\ell}$, but the discovery reach in the combined production mode [7] can reach the TeV scale and the sensitivity to $\lambda_{e}$ can be considerably improved over present limits from LEP [8].

At the $\mathrm{LHeC}$, not only can the mass reach be improved [9], but also many of the quantum numbers and couplings can be measured. In particular, the LQ fermion number $F$ can be obtained $[10,11]$ from the asymmetry parameter $A=\left(\sigma_{e^{-}}-\sigma_{e^{+}}\right) /\left(\sigma_{e^{-}}+\sigma_{e^{+}}\right)$, where $\sigma_{e^{ \pm}}$is the cross section in $e^{ \pm}+p$ production channel. This asymmetry, which is due to the fact that valence quarks in the proton have a higher $x$ than sea antiquarks, is independent of the coupling $\lambda_{e}$ since this term cancels out in the ratio. It must be noted that, although a similar asymmetry can also be measured at the LHC, the sensitivity is lower there because of the lower cross section for single production. This asymmetry term is also sensitive to the charge of the LQ, as can be seen in Fig. 1(left). Information on other quantum numbers can also be obtained at the LHeC: the scalar or vector nature can be determined from the angular distribution of the electron from LQ decay with respect to the initial beam direction, in the rest frame of the LQ, as is illustrated convincingly in (Fig. 1,right); their chirality (i.e. whether they couple to Left-handed, Right-handed quarks only, or to both types) can eventually be probed by measuring the sensitivity of the cross section to the polarization of the electron beam; the possibility of generation mixing could be investigated by searching for $\mu$ or $\tau$ in the final state; the branching ratio to neutrino can be determined by searching for the appropriate decay channel, e.g. $e_{L}^{-} u_{L} \rightarrow S_{3} \rightarrow v_{e} d_{L}$, where $S_{3}$ is a scalar isodoublet LQ 
of charge 1/3, following Buchmüller's classification [12]. Both Figs 1 were obtained with a LQ model [13] in CalcHep [14].
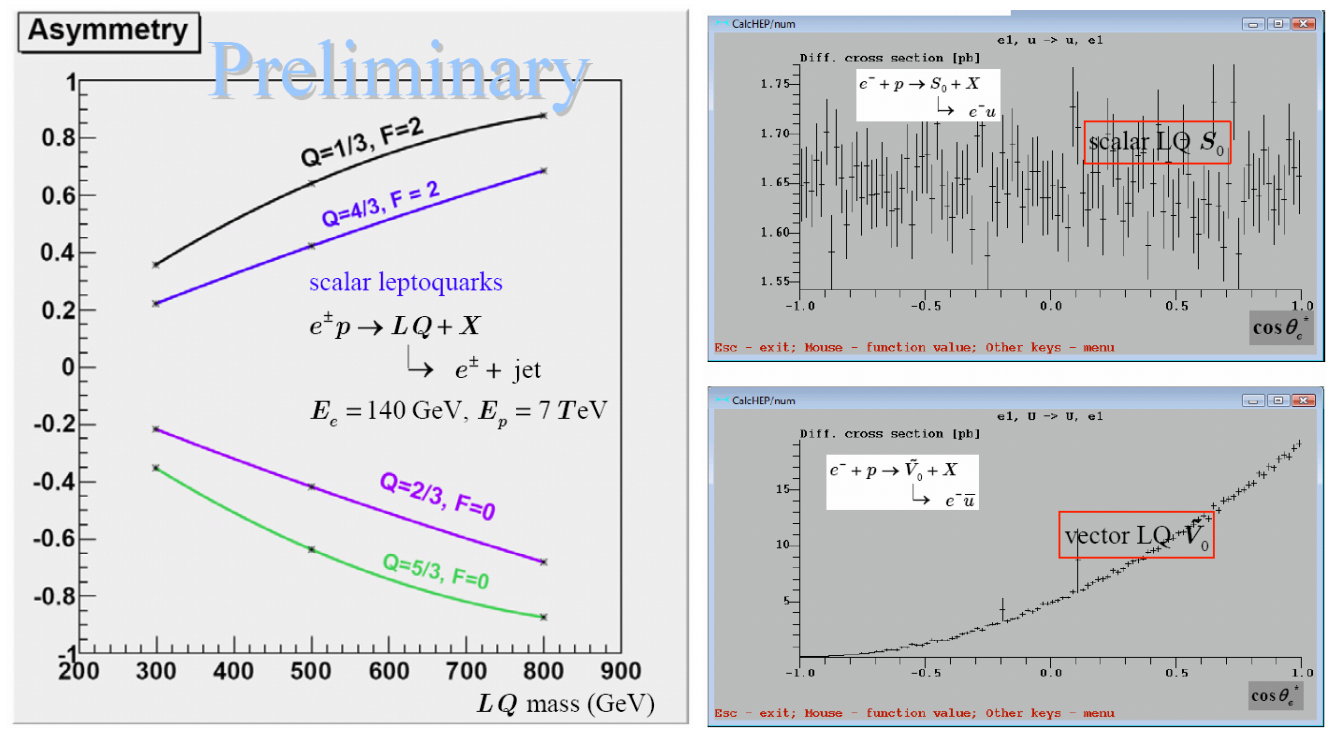

Figure 1: left: Charge asymmetry vs LQ mass for different types of scalar LQ's. right: angular distribution of the LQ decay in its rest frame, with respect to the direction of the electron beam, for a scalar and a vector LQ.

\section{Contact Interactions}

If new physics occurs at a scale $\Lambda \gg \sqrt{s}$, then it will appear as an effective contact interaction with coupling of order $1 / \Lambda^{2}$ between fermion currents $j_{\mu}^{(f=e, q)}=\eta_{L} \bar{f}_{L} \gamma_{\mu} f_{L}+\eta_{R} \bar{f}_{R} \gamma_{\mu} f_{R}+$ h.c. and limits could be obtained on the different coupling combinations $\eta_{i j}=\eta_{i}^{(e)} \eta_{j}^{(q)}$. For example, at $\sqrt{s}=140 \mathrm{GeV}$, with $20 \mathrm{fb}^{-1}$, sensitivity to scales $\sim 30-40 \mathrm{TeV}$ can be obtained [9] for parity conserving or parity violating combinations of couplings, which is an improvement by about a factor 5 over HERA results. If interpreted in terms of models of large extra dimensions, effective Plank mass scales up to about $5.4 \mathrm{TeV}$ can be probed.

\section{Heavy and Excited Leptons}

In composite models, fermions can be made up of more fundamental constituents. Excited fermions could then be produced at energies close to or above the compositeness scale via contact or gauge interactions. Since the contact interaction cross section varies as $1 / \Lambda^{4}$ whereas gauge interaction cross section varies as $1 / \Lambda^{2}$, the former mode is less significant at high mass. In ref [15], it is shown that the LHeC could extend the reach for excited electrons, in the $e^{*} \rightarrow e \gamma$ decay mode. For example, at $\sqrt{s}=1.9 \mathrm{TeV}$, with $1 \mathrm{fb}^{-1}$, a mass of up to $1.4 \mathrm{TeV}$ can be discovered, assuming $f / \Lambda=f^{\prime} / \Lambda=1 / m_{e^{*}}$, where $f, f^{\prime}$ are factors multiplying the SM $S U(2)_{L}$ and $U(1)$ gauge couplings.

Heavy fermions can also exist as a fourth SM family, or in extended gauge theories such as $E_{6}$ [2], or just as vector-like fermions. Although they would be copiously produced at the LHC, 
the measurement of anomalous coupling to SM fermions would be difficult. At the $\mathrm{LHeC}$, with $\sqrt{s}=1.4 \mathrm{TeV}$ and $1 \mathrm{fb}^{-1}, 5 \sigma$ discovery of heavy charged leptons [16] can be achieved up to a mass of $800 \mathrm{GeV}$, assuming $\kappa_{\gamma}^{e} / \Lambda=\kappa_{Z}^{e} / \Lambda=1 \mathrm{TeV}^{-1}$. Similar results can be obtained for charged current production of fourth family neutrinos [17].

\section{Heavy and Excited Quarks}

At the $\mathrm{LHeC}$, single production of $4^{\text {th }}$ family up- or down-type quarks via charged current interactions can compete with LHC measurements, where background is likely to be higher [18, 19]. Such production depends on the extended $4 \times 4$ CKM matrix elements which are strongly constrained.

In the $\gamma p$ mode of operation of the $\mathrm{LHeC}$, anomalous production of top quarks can constrain the couplings $\kappa_{c}^{\gamma}$ and $\kappa_{u}^{\gamma}$, leading to an improvement over the LHC for the branching ratio $B R(t \rightarrow$ $u \gamma)$ by two orders of magnitude [20]. In this same mode of operation, for a fourth family $t^{\prime}$, assuming only anomalous couplings to lighter generations and $\kappa_{g}=0$, bounds on $\kappa_{\gamma} / \Lambda$ and $\kappa_{Z} / \Lambda$ can reach a fraction of $\mathrm{TeV}^{-1}$ [21].

Finally, as another example, diquarks, which can also be present in $E_{6}$ models [2] can also be investigated in the $\gamma p$ production channel, achieving a discovery reach of $900 \mathrm{GeV}$ for a machine of $140 \times 7000 \mathrm{GeV}^{2}$ and with $\alpha_{D Q}=0.1$ [22].

\section{Conclusion}

The remarkable symmetries observed between the lepton and quark sectors cannot be a coincidence and must surely derive from a fundamental relation between them. This relation can take many different forms such as the presence of leptoquarks or lepton-quark compositeness. The examples given above of physics beyond the Standard model demonstrate clearly that the LHeC is perfectly suited to probe this relation at the TeV scale. Results will complement the LHC in understanding the new phenomena and a more complete interpretation of the LHC discoveries will be possible.

\section{References}

[1] The LHeC: Deep Inelastic Electron-Nucleon Scattering at the LHC, M. Klein, convenor http: / / www.ep.ph.bham.ac.uk/exp/LHeC/.

[2] J. L. Hewett and T. G. Rizzo, Low-Energy Phenomenology of Superstring Inspired E(6) Models, Phys. Rept. 183 (1989) 193.

[3] E. Farhi and L. Susskind, Technicolor, Phys. Rept. 74 (1981) 277.

[4] C. T. Hill and E. H. Simmons, Strong dynamics and electroweak symmetry breaking, Phys. Rept. 381 (2003) 235-402, arXiv: hep-ph/0203079.

[5] J. C. Pati and A. Salam, Lepton Number as the Fourth Color, Phys. Rev. D10 (1974) 275-289.

[6] The ATLAS Collaboration, G. Aad et al., Expected Performance of the ATLAS Experiment - Detector, Trigger and Physics, arXiv:0901.0512 [hep-ex]. 
[7] A. Belyaev, C. Leroy, R. Mehdiyev, and A. Pukhov, Leptoquark single and pair production at LHC with CalcHEP/CompHEP in the complete model, JHEP 09 (2005) 005, arXiv : hep-ph / 0502067.

[8] Particle Data Group Collaboration, C. Amsler et al., Review of particle physics, Phys. Lett. B667 (2008) 1. (http://www-pdg.lbl.gov/) .

[9] A. F. Zarnecki, Leptoquarks and Contact Interactions at LeHC, arXiv:0809.2917 [hep-ph ] .

[10] E. Perez, Physics beyond the standard model at LHeC, . Prepared for 15th International Workshop on Deep-Inelastic Scattering and Related Subjects (DIS2007), Munich, Germany, 16-20 Apr 2007 (http://www.sciwipub.com/proceedings/DIS2007/perez_emmanuelle.pdf).

[11] E. Perez, Physics Opportunities at the LHeC, at From the LHC to a Future Collider, Feb 2009, CERN, (http://indico.cern.ch/conferenceDisplay.py?confId=40437).

[12] W. Buchmuller, R. Ruckl, and D. Wyler, Leptoquarks in lepton quark collisions, Phys. Lett. B191 (1987) 442-448.

[13] A. Belyaev and A. Pukhov. private communication.

[14] A. Pukhov, A. Belyaev, and N. Christensen, CalcHep, http://theory.sinp.msu.ru/p̃ukhov/calchep.html.

[15] T. Nguyet Trinh and E. Sauvan, First ECFA-CERN LHeC Workshop, Divonne 2008, (http://indico.cern.ch/conferenceDisplay.py?confId=31463).

[16] A. K. Ciftci, R. Ciftci, H. Duran Yildiz, and S. Sultansoy, Anomalous single production of the fourth generation charged leptons at future e p colliders, Mod. Phys. Lett. A23 (2008) 1047-1054, arXiv:hep-ph/0612215.

[17] A. K. Ciftci, R. Ciftci, and S. Sultansoy, Anomalous Single Production of the Fourth Generation Neutrino at Future ep Colliders, Phys. Lett. B660 (2008) 534-538, arXiv: 0711.4153 [hep-ph].

[18] O. Cakir, A. Senol, and A. T. Tasci, Single Production of Fourth Family $t^{\prime}$ Quarks at LHeC, Europhys. Lett. 88 (2009) 11002, arXiv:0905.4347 [hep-ph].

[19] O. Cakir and V. Cetinkaya, Single Production of Fourth Family b' Quark at the Large Hadron electron Collider, arXiv:0912.2041 [hep-ph].

[20] I. T. Cakir, O. Cakir, and S. Sultansoy, Anomalous Single Top Production at the Large Hadron electron Collider Based gamma p Collider, Phys. Lett. B685 (2010) 170-173, arXiv: 0911.4194 [hep-ph].

[21] R. Ciftci and A. K. Ciftci, A Comperative Study of the Anomalous Single Production of the Fourth Generation Quarks at ep and gamma-p Colliders, arXiv:0904.4489 [hep-ph] .

[22] M. Sahin and O. Cakir, Search for Scalar Diquarks at the LHeC Based Gamma-Proton Collider, arXiv:0911.0496 [hep-ph]. 